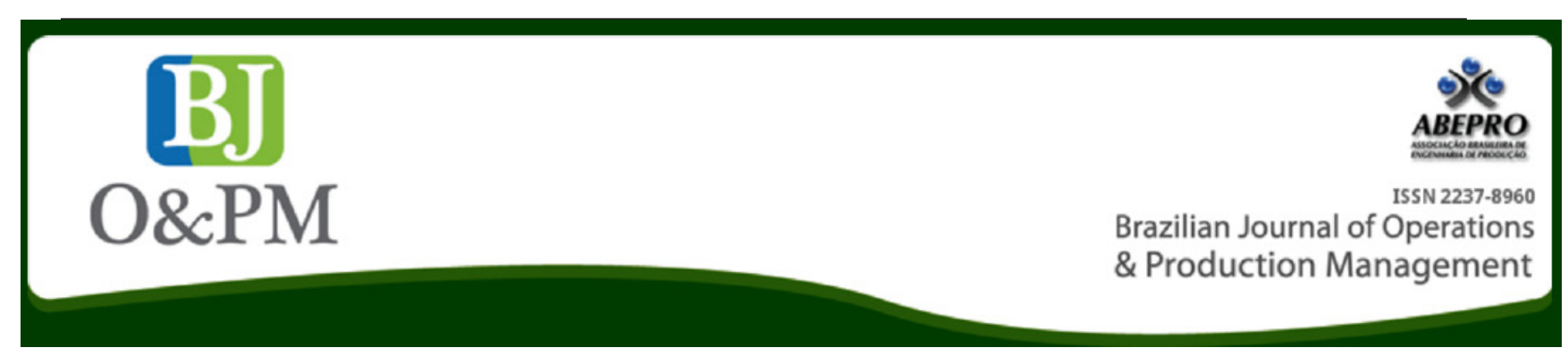

\title{
UTILIZATION OF FUZZY CONSTRAINTS TO BUILD APPLICATIONS TO SUPPORT A CONCURRENT ENGINEERING ENVIRONMENT IN THE PROCESS OF DESIGN AND MANUFACTURING
}

\author{
Rubens Aguiar Walker ${ }^{a}$, Sergio Baltar Fandiño ${ }^{b}$, Alexandre Camacho Paixãoc, Marlene Jesus Soares Bezerra ${ }^{b}$ \\ a University of Rio Grande (UNIGRANRIO) - Rio de Janeiro, RJ, Brazil \\ ${ }^{b}$ Foundation State University Center of the West Zone (UEZO) - Rio de Janeiro, RJ, Brazil \\ ' Estacio de Sa University (UNESA) - Rio de Janeiro, RJ, Brazil
}

\section{ABSTRACT}

This article proposes an integration between Conceptual Design and Manufacturing stage through a Fuzzy set application. In the beginning of the ideas the set of values does not include a risk for the project and if it is able to do it or not. In this case it basically setting a data-model thought what is big, small, high quality, expensive, and others values, with the real manufacturing data as fuzzy numbers. As the product is developed a decision can be easy make as the capability of manufacturing with an idea of quality and cost. In logistic relate the data with small, medium and high volume, combining with delivery data range. Since this application is a related proposed data set, it can increase the capability with new available data. Supporting a Concurrent Engineering environment to integrate and validate the decision.

Keywords: Design, Manufacturing, Fuzzy 


\section{INTRODUCTION}

Since the 80 's Concurrent Engineering is a technique that has been used to reduce the time-to market for a new product. Concurrent Engineering differs from classical approaches in that it moves the sequential flow of product develops to a parallel process by overlapping the activities. The basic idea is integrated product and process development, Sweetman 2000.

This paper describes a computer application using fuzzy logic constraint to predict values of design in a Concurrent Engineering view.

\section{CONCURRENT ENGINEERING}

There are two approaches for the Concurrent Engineering inside the enterprise, Sobek II 1999, 'human': make cross functions times, benchmarking; and 'computer': Design for Manufacturing \& Assembly Quality Function Deployment, Robust Design, integrated CAD, Simulation, Product Data Management, etc.

The human approach is based in collaborative groups to develop a project. This means in the creation of cross functions teams using at least one person of each stage of the design, process planning, manufacturing, and others like logistic and sales. The cross function time has the responsibility to link the ideas of the product development through the capability of the factory to over lap the functions in the different stage of the new project. Some studies of this model give a limitation for the capability of developing the project with a complete integration. This means that the higher complexity of the product and the manufacturing the more time you spend to communicate among the times. To simplify the model some subdivision can be made to a modular interfaces of the product and/or improve the information technology, Schmitz 1999.

The computer approach is based on CAD systems that interact through the design stages. Many CAD's systems can be manager for the final result of the project as 3D CAD model, used to develop the preliminary stage of the design, and 2D CAD documentation, used to develop the detail stage of the design. The data usually are manager with a product data management (PDM). Usually a UNIX platform is used to $3 D$ CAD model and widows platform to $2 D$ CAD documentation. For a final manufacturing documentation usually make by a CAPP system for the manufacturing path of the design.

In general this two approach have developed in different scale of time, the short-term and the-long term. The shortterm is recognized by the reduce of the time-to-market and the higher quality of the product in the beginning of the implementation of the CE. The transition of the skills for other project take some time to adjust the method to the requirements of the organization. In this long term the product develop team may have some problem in identify lower priority items and lose a bunch of time in the process of make a decision, Fleischer 1999.

The supply change can be considered a third approach. In this case the integration between the enterprises is critical for the knowledge of each enterprise limits. A supply change must be considered a partner and develop the new products together. The communication is very important to set the design development. In this case the transparence of the manufacturing and its limits are very important to make the decisions for develop a new product Schmitz 1999.

Toyota makes a successful in CE in develop many small prototypes, a strong organization of the activities without the necessity of a multifunctional team to make small decisions, and just do the final choice at the end. In this case they avoid in given up of some good solutions on the process of develop the new project, Hoedemaker 1999.

The CE brings many changes in the way to see the flow of the product development and become a routine practice, Fleischer 1999, but most of the time the enterprises do not need all the changes or can make its gradually.

Most of the bibliography speaks about the integration of the product, but a critical factor is to predict values to make a decision during the process of develop the design. This paper describes how we can use fuzzy constrains to predict values.

\section{DESIGN AND MANUFACTURING ENVIRONMENT}

Not many products are new or innovative that actually belong to the manufacturing platform of the enterprise. Most of the information can be explained through knowledge and linkages between different design stages. The design is represented by six stages - the conceptual, the embodiment, preliminary, detail, process planning, and manufacturing. The conceptual stage is responsible for the ideas and functions of the product design. The embodiment stage represents the details of the geometric features and manufacturing. The detail design stage is the documentation of the project. The process planning stage is developing the product fabrication and assembly. The manufacturing stage is the final stage that aggregates the all information of the design to make a product, Varley 1999.

The planning and design process is divided into the following phases: product planning and clarifying the task, conceptual design, embodiment design, and detail design. The working steps proposed for each of the main phases must be considered the main working steps. The results of these main working steps provide the basis for the subsequent ones. Some results in many lower levels 
working steps are required such as collecting information, searching for solutions, calculating, drawing and evaluating. Each of these working steps are accompanied by indirect activities such as discussing, classifying and preparing.

Before a commercial product can be designed there has to be a product idea, which promised to lead to a technically and economically viable product. In many companies the product planning department is expected to follow the development of product idea through the design and manufacturing departments, and then to watch over this market behavior. This includes monitoring the financial position and market success of the product and if necessary, taking appropriate corrective measures. Companies deal with product planning in different ways, in many cases is a good sense of a director, or an individual employee. However, systematic procedures are frequently used to find new ideas, that are easier to measure the time and the cost of product planning and development. The basis and beginning point is marketing, that provides an interface between customer and product planning. The internal and external stimuli for product plan come from marketing, the company, and others sources. Those give us five main working steps: Analyzing the Situation, Formulating Search Strategies, Finding Products Ideas, Selecting Products Ideas, and Defining Products, Teschler 1999.

The Conceptual Design consists in identifying the essential problem through abstraction, by establishment of function structures and by the search for appropriate working principles and their combination, the basic solution path is down through the elaboration of solution principles.

The Embodiment Design must determinate the overall design (general arrangements and spatial compatibility), the preliminary from design (components shapes and materials) and the production process, and provide solutions for any auxiliary functions.

The Detail Design completes the embodiment of technical products with final instructions about the layout, forms, dimensions, and surface proprieties of all individual components, the definitive selection of material and a final scrutiny of the production methods, operating procedures and cost. In this phase it is responsible to elaborate the production documents, especially of detailed components and assembly design and of appropriate parts list.

The Process Planning is a role in detailed procedures by which work pieces or parts are converted from the initial stage (raw material form) to finished stage (desire form). Product design data such as geometrical features, dimensional sizes, tolerances, material, and finishes are analyzed and evaluated to determine the appropriate workstation available within the manufacturing systems concerned. Those detail methods are then documented into process plane or processes sheets, which server as technical instructions on the shop floor, and assure the desired characteristics of the final product (Grove, 2006).

The Manufacturing is understood as a series of activities including customer need recognition, design, material selection, planning, material transformation process, quality assurance, and logistic management. Basic means the material transformation processes through machines, tolls, and any device to get the design model. Manufacturing is responsible to do or build the product, including fabrication of part number and assembly. Some critical conditions are dealt with tolerance a finishing for transformation the material through the available machines.

The environment of computer applications that aid concurrent engineering are CAD 3D, CAD 2D, CAPP, CAM, expert systems, etc. In this case, we link the product features between different stages of development. So in each stage we can have more detail of the design and can represent its limits and anticipate some design values for the project development. At the same time, we do not need to detail all the features of the project for documentation. In this case the results of this information can be used to start the documentation of the design in a CAD system and the process planning in a CAPP system.

\section{PROBLEM}

The enterprise is a subsidiary in Brazil, and consists in sales hydraulic pumps by order and standard. In spied of be by order the parts number are standard to the customer. The factory does not need to develop a conceptual design of a new product, but just make some alterations to attend specifications or material changes of marketing. This made constant consult to preliminary evaluation of the viability design cost.

It does not exist multifunctional times. Usually the employee change is because someone gets out of the enterprise. Chefs trust in the individual experience of each employee to develop embodiment and detail design. The knowledge pass is not formal trough the enterprise or the employee. This area is extremely pressed what makes it difficult to interchange design and manufacturing functions, see figure 1.

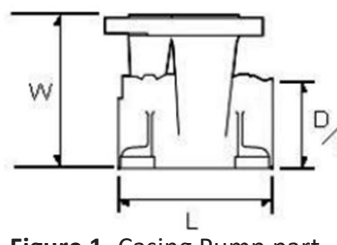

Figure 1. Casing Pump part

Source: The author own

The enterprise has a good computer automation degree in the embodiment and detail design phases. These represented by $3 D C A D$ to model and $2 D C A D$ to 
documentation and a CAPP system to integrate to the manufacturing. It does not have any expert system to integrate and validate functions. The information stream is aided by a Work Group system that can used to pass Email and mandatory actions.

There are many numerical control (NC) machines associated to a machine center. There is also an effort to standardization the information part number in families that is represented by the Group Technology.

The only pointer of performance is the manufacturing quality measure. There is no point performance to the design or any decisions make.

The Research \& Development sector is located in USA. The research is represented by under graduation final project developed by trainees.

\subsection{What are the problems?}

- In the description of the industry engineer you can find some problems, it is not so difficult to compare with the necessity in integrate function to get in the Concurrent Engineering environment, there are:

- No multifunction times;

- No integration between design phases and manufacturing process;

- No integration between the systems CAD 3D, CAD 2D, CAPP, CN program;

- No system to analyze cost with changes in the design or manufacturing phases;

- No formal documentation of the knowledge;

- No complete standardization of the information to use Group Technology;

\subsection{What are the goals?}

- Integration of information between embodiment design, detail design, process planning, and manufacturing phases trough a system;

- A system that helps in costs analysis in changes of design, process planning and manufacturing;

- Data standardizing;

The researched enterprise environment has presented some problems and difficulties in engineering. This can be identified as necessities of integration that are present in Concurrent Engineering. For example: a company does not have any multifunctional teams, any integration between the design and manufacturing stages, does not have any system to analyze cost and viability in changes design parameters, etc. To help address these problems we model an application which is explained later in this article.

\section{AI AND FUZZY LOGIC}

Throughout the enterprise environment there is inherent imprecision and soft specifications represented by cost, quality and dimensionality. Soft specifications are more prevalent in the early stages of the design and within manufacturing in its use of tolerances, imprecision is more prevalent. We use fuzzy data to model the inconsistency and imprecision. This helps the designer to develop the idea of the design project without the necessity of precise specifications and values, figure 2 . Later, some information can be more precise in other design stages while other information must still deal with imprecision in the parameters in the fabrication and assembly environment. This is the imprecision of manufacturing tolerances and can be better described using a fuzzy numbers.

The description matches the distributed processing environment in a fuzzy constraint system. The applications are built in the FUZCON (Fuzzy Constraint Processing System), MS-Access database, and using Delphi to build the user interface. It is divided into four modules: conceptual design, embodiment design, processes planning, and manufacturing. All these modules exchange information among themselves and with different databases. All the data are used for manufacturing components of a hydraulic pump.

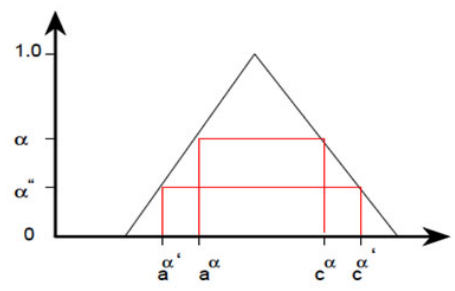

Figure 2. Fuzzy Constraint Source: Giachettiung , 1998

You can vary the value of the variable for the project acceptance. For example some degree can vary between a range to be acceptable, but for somebody else this range can be more or less compared to yours.

\section{SYSTEM}

The emphasis of this paper is describing a computer application developed to predict values. The example is made on the development of parts of a hydraulic pump. The development stages of the design are conceptual, embodiment, process planning, and manufacturing. In the conceptual stage the designer just have an idea new product, the quality, and the dimensions, some information about the cost will be very helpful to make decisions in this early stage. In the embodiment stage of the design the designer define the geometric features, the material, and some specifications. The process planning stage document 
the manufacturing process and optimize the manufactory process cost. The manufacturing stage of the product is the machines and tools of job shop, represented through its features in a database.

It was made a computer application divide in four modules as: Conceptual Design, Embodiment Design, Process Planning, and Manufacturing.

The Conceptual Design is the first module that represents the conceptual of the generically idea of the product, dimension, geometry, quality, and cost. In this phase the designer just has an idea of what he will do. Although this information is not enough to compare with the set manufacturing features but it can give us some early parameters of quality, dimension, and cost. With the notion of low, medium, high, or standard, the designer can set some relations and some values for the constrains. Crispy and fuzzy numbers set generic associations for the next stage of the design. It guarantees some relations as high quality, big dimension with low cost cannot happen.

The Embodiment Design represents the second stage or module. The design set the geometry and its features, the material, the tolerance, and the finish, according with the sets of features in the conceptual design.

In this module the cost does not restrict the others domain values. The design features are represented by principal geometric features as diameter, length and width. In the future the design feature can be integrated with a CAD system. The manufacturing features (hole, flange, cut) are chosen by designer and restrict the process and the manufacturing. These values are essential to evaluate the manufacturing and the cost.
The restriction represented by dimensions and quality in the domain for the geometry, the finish, the tolerance, and the centricity, that has fuzzy data. In this case the propagation of the constraint is through restrictions and ID avoiding error propagation in the fuzzy numbers, Young et al., (1996). To get a better efficient and avoid making many objects that are represented by the design and manufacturing features an interface with the user has been constructed. In this case the final user does not need many objects, but only choose one time. This way we simplify the procedure and at the same time do not restring the number of objects. The set of values is propagated to process planning and manufacturing modules, on figure 3 .

The Process Planning module describes the manufacturing process for the component or product. In this case it is going to show the time in each operating that is in the database. This data will be used to calculate the manufacturing cost and can be represented by fuzzy numbers.

To recognize the manufacturing process through the manufacturing feature object, set in the Embodiment Design, direct related using ID for the process planning operation and each respective time.

In some cases the user can choose alternatives process to reduce cost or substitute process.

Manufacturing module is represented by a database of machines and its respective tools. In this application it compares the geometry, tolerance, concentric, and finish fuzzy data according with the sets in the Conceptual Design module of the truth value. When the machines are all set trough the component we can know the value of the cost/ hour to calculate the final cost of each component. With the

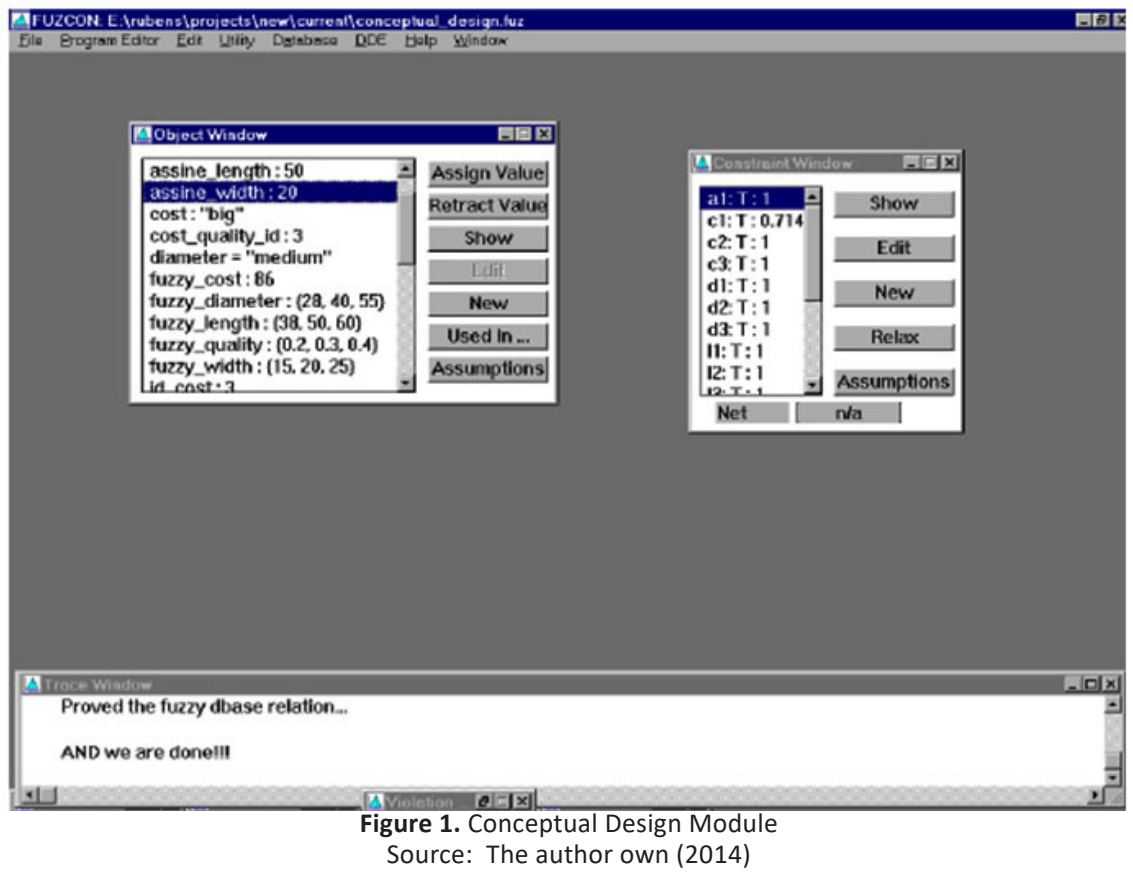


machines and components in the database we can set the manufacturing cells, according win the volume, dimension, and cost. In same case we can add a machine and see if possible to reduce the cost of manufacturing for different components.

\subsection{Example}

The designer is checking the possibility of made a pump house. To solve it the designer must give for this component a proportional dimension (ex. Big), quality (high), and the system generate a high cost solution for this specific case. In others cases that you have more than one solution you must set the value to see if is possible or not. The next stage is representing the dimensions and quality values for geometry and manufacturing features in the embodiment design that is automatic restrict the domain by conceptual when choose, 'enormous', 'big', 'medium', 'small', or 'standard'. When the embodiment designer chooses the manufacturing features as: through hole, assembly guides hole, surface glides, etc., it propagates the relation and defines the process planning and the possible machines with its set of tools. The cost, the tolerance, and the finish, as the dimension restrict set of machines. The sum of process time plus cost/hour of each machine calculate the cost.

When the tolerance is higher the application can accept the solution if the respective truth-value is significant in the range of fuzzy numbers. In this case some solutions that are usually ignore, can be significant in the process optimize cost or give some alternatives for the manufacturing.

In the illustration we can see a representation for the computer application in the "Conceptual Design" for the upper pump.

\subsection{Results}

The expected results of the computer application involve three goals. The first, demonstrate integration of information between conceptual design, embodiment design, detail design, process planning, and manufacturing stage through a prototype system. The second, a system that helps in cost analysis when changes are made in design, process planning and manufacturing. The third, use fuzzy constraints to model data inconsistency. The data at each design stage is used to determine the viability of the project and its cost.

\section{CONCLUSION}

This research expects to develop a computer application that helps in the making decision of a designer, process planner or manufacturing engineer. In each stage we validate the information and anticipate others such as cost or quality. The expected results and the industry summarize the interests of each author in building applications to aid the design and manufacturing using fuzzy constraint. In this approach the link and the anticipation of the data values helps the Concurrent Engineering environment to deal with different possibilities for the design and manufacturing.

\section{REFERENCES}

Fleischer, M. (1999), "CE in the long run"; Mitchell; Automotive Manufacturing \& Production,

Cincinnati; Jan; Vol. 111, Iss. 1; pg. 14, 1 pgs .

Giachetti, R. E.; Young, R. E. (1998), "Analysis of the Error in the Standard Approximation Used for Multiplication of Triangular and Trapezoidal Fuzzy Numbers and the Development of a New Approximation", Fuzzy Sets and Systems.

Groove, M; "Automation, Production Systems, and Computer-Integrated Manufacturing" $3^{\text {rd }}$

Hoedemaker, G. M. (1999), "Limits to currency"; Decision Sciences, Atlanta; Winter; Vol. 30, Iss. 1; pg. 1, 18 pgs .

Pahl, G. and Beitz, W. (1997), 'Engineering Design"; London, Ed. Springer, 543 p.

Prentice Hall Press Upper Saddle River, NJ, USA C)2007

Schmitz, B. (1999), "Half the time, half the cost"; Computer - Aided Engineering, Cleveland; Vol. 18, Iss. 5; pg. 9, 1 pgs .

Sobek II, D K. (1999), "Toyota's principles of set-based concurrent engineering"; Sloan Management Review, Cambridge; Winter; Vol. 40, Iss. 2; pg. 67, 17 pgs.

Sweetman, D. (2000), "What makes a winner?" Beverage World, New York; Vol. 119, Iss. 1693; pg. 56, 2 pgs.

Teschler, L. (1999), "CE's best practitioners"; Machine Design, Cleveland; Vol. 71, Iss. 13; pg. 76,5 pgs

Varley, P. (1999), "Plane thinking"; Supply Management, London; Vol. 4, Iss. 5; pg. 39, 2 pgs.

Young, R. E.; Giachetti, R. E.; Ress, D. A. "A Fuzzy Constraint Satisfaction System forDesign and Manufacturing,", FUZZIEEE'96 Proceedings, vol. II, , pp. 1106-1112 FUZZ-IEEE'96, vol. II, 1996. 